\title{
Fatores associados à atividade física e aos comportamentos sedentários em adolescentes
}

\author{
Physical activity and sedentary behaviors associated \\ factors in adolescents
}

Daniel Giordani Vasques
Adair da Silva Lopes

1 Universidade Federal de Santa Catarina. Programa de Pós-Graduação em Educação Física, Bolsista CNPq. Florianópolis, SC. Brasil

2 Universidade Federal de Santa Catarina. Departamento de Educação Física. Programa de PósGraduação em Educação Física. Florianópolis, SC. Brasil.

Recebido em 30/12/07 Aprovado em 14/07/08
Resumo - Objetivou-se analisar fatores associados à atividade física e aos comportamentos sedentários em adolescentes. A amostra foi composta de 1675 escolares (784 rapazes e 891 moças), de 11 a 17 anos de idade, de Caxias do Sul-RS. Um questionário foi aplicado para identificar o nível de atividade física (recordatório de 3 dias) e o número de horas em comportamentos sedentários. Considerou-se como baixo nível de atividade física (baixo NAF) um gasto energético menor do que $37 \mathrm{kcal} /$ $\mathrm{kg} /$ dia e exposição elevada a comportamentos sedentários (CSE) assistir TV, jogar videogame ou utilizar computador mais do que $14 \mathrm{~h} /$ semana. Os resultados do teste qui-quadrado indicaram que as moças apresentaram maior prevalência de baixo NAF $(66,8 \%>43,2 \% ; p<0,001)$, enquanto os rapazes obtiveram maior prevalência de CSE $(89,6 \%>84,0 \% ; p=0,001)$. Baixo NAF associou-se aos rapazes a partir da regressão de Poisson, com residir com até 4 pessoas ( $\mathrm{RP}=1,21 ; \mathrm{IC} 95 \% 1,00-1,46)$ e com o baixo NAF da mãe (RP=1,23;IC95\%1,00-1,53), e nas moças, com a faixa etária de 15-17 anos (RP=1,30;IC95\%1,18-1,44), residir com até 4 pessoas ( $\mathrm{RP}=1,17 ; \mathrm{IC} 95 \% 1,04-1,31)$, possuir $\mathrm{TV}$ no quarto $(\mathrm{RP}=1,13 ; \mathrm{IC} 95 \% 1,02-1,25)$ e deslocar-se passivamente à escola ( $\mathrm{RP}=1,10 ; \mathrm{IC} 95 \% 1,00-1,22)$. A CSE associou-se às moças à maior escolaridade do chefe da família $(\mathrm{RP}=1,08 ; \mathrm{IC} 95 \% 1,01-1,16)$ e a possuir TV no quarto $(\mathrm{RP}=1,15 ; \mathrm{IC} 95 \% 1,08-1,22)$. Destaca-se a associação entre as medidas de aproximação socioeconômica e os comportamentos de risco (baixo NAF e CSE). O conhecimento dos fatores associados ao baixo NAF e à CSE facilita a implantação de intervenções mais efetivas, a fim de promover um estilo de vida mais ativo.

Palavras-chave: Atividade física; Comportamento sedentário; Saúde do adolescente.

Abstract - The purpose of this study was to analyze factors associated with physical activity and sedentary behaviors in adolescents. The sample consisted of 1675 students (784 boys and 891 girls) ranging in age from 11-17 years from Caxias do Sul, RS. A questionnaire was applied to identify physical activity level (PAL, 3-day recall) and weekly hours of sedentary behavior. Low PAL was defined as energy expenditure less than $37 \mathrm{kcal} / \mathrm{kg}$ per day, and elevated sedentary behavior (ESB) was defined as more than $14 \mathrm{~h} /$ week watching TV, playing video games or using a computer. Chi-square test results indicated a higher prevalence of low PAL among girls $(66.8 \%>43.2 \%, p<0.001)$, whereas the prevalence of ESB was higher among boys $(89.6 \%>84.0 \%, p=0.001)$. Using a Poisson hierarchical regression model, low PAL was associated with up to 4 persons living at home (PR=1.21,95\%CI: $1.00-1.46)$ and low maternal PAL $(P R=1.23,95 \% \mathrm{CI}$ : 1.00-1.53) among boys, and with age 15-17 years (PR=1.30, 95\%CI: 1.18-1.44), up to 4 persons living at home (PR=1.17, 95\%CI: 1.04-1.31), having a TV in the bedroom $(P R=1.13,95 \% \mathrm{CI}: 1.02-1.25)$ and passive transport to school $(P R=1.10,95 \% \mathrm{CI}: 1.00$ 1.22) among girls. In girls, ESB was associated with high parental educational level $(P R=1.08,95 \% \mathrm{CI}: 1.01-1.16)$ and having a TV in the bedroom $(P R=1.15,95 \% \mathrm{CI}$ : 1.08-1.22). The results suggest an association between socioeconomic measures and risk behaviors (low PAL and ESB). Knowledge about the factors associated with low PAL and ESB facilitates the implantation of more effective interventions in order to promote a more active lifestyle.

Key words: Physical activity; Sedentary behavior; Adolescent health. 


\section{INTRODUÇÃO}

A manutenção de níveis adequados de atividade física na adolescência é um fator componente de um estilo de vida saudável ${ }^{1}$, à medida que promove benefícios à saúde óssea e cardiovascular, além de estar associado à manutenção da atividade física na fase adulta ${ }^{2}$. Entretanto, as prevalências elevadas de sedentarismo (de $41,6 \%^{3}$ a $95 \%{ }^{4}$ em municípios brasileiros) e a associação destas com fatores de risco para morbidades ${ }^{5-7}$ indicam a importância de intervir quando a prática de atividade física encontra-se abaixo dos níveis adequados.

Para o desenvolvimento de programas de intervenção em atividade física para adolescentes é necessário o conhecimento dos fatores associados à atividade física. Dados de associação de estudos transversais podem ajudar a identificar grupos de risco com baixos níveis de atividade física a serem focados em intervenções ${ }^{8}$. Estudos têm mostrado que as prevalências de baixos níveis de atividade física tendem a ocorrer em maior grau nos adolescentes do sexo feminino, que realizam deslocamento passivo para a escola $^{10}$,cujos pais possuem baixos níveisl de escolaridade e de atividade física ${ }^{8,9}$.

No entanto, foram poucos os estudos em adolescentes brasileiros que analisaram conjuntamente fatores associados à atividade física e aos comportamentos sedentários. Sendo assim, o objetivo deste estudo foi analisar fatores associados à atividade física e aos comportamentos sedentários em adolescentes de Caxias do Sul, RS.

\section{PROCEDIMENTOS METODOLÓGICOS}

Este estudo caracterizou-se como epidemiológico de corte transversal e é parte do projeto "Fatores de risco para doenças cardiovasculares em adolescentes de Caxias do Sul, RS, Brasil", aprovado pelo Comitê de Ética para Pesquisas com Seres Humanos da Universidade Federal de Santa Catarina, sob o protocolo 078/2007. A coleta de dados ocorreu de maio a julho de 2007, em escolas de Caxias do Sul, RS, município da região nordeste do RS, que possui aproximadamente $420 \mathrm{mil}$ habitantes e o $12^{\circ}$ maior Índice de Desenvolvimento Humano (IDH=0,857) do Brasil.

Para o cálculo do tamanho da amostra, utilizou-se a proposta de Luiz e Magnanini ${ }^{11}$, a partir de um erro amostral de três pontos percentuais. Considerou-se a prevalência de sedentarismo, onde estudos com adolescentes de municípios do sul do Brasil apontam ser de aproximadamente $60 \%{ }^{12,13}$ e foi utilizado um efeito de delineamento de 1,5. Encontrou-se um número mínimo de 1500 adolescentes e, somando 20\% para compensar possíveis perdas e recusas, o cálculo foi de 1800 sujeitos. Foram contatados 1835 escolares, sendo que 54 recusaram-se a participar da pesquisa ou não retornaram o questionário. Excluíram-se os que não preencheram o sexo e/ou a idade $(n=15)$. Não fizeram parte da amostra (perdas) os adolescentes que possuíam idade inferior a 11 anos $(n=62)$ e igual ou superior a 18 anos $(\mathrm{n}=29)$, totalizando 1675 escolares.

Embora o tamanho da amostra tenha sido calculado a partir da prevalência de sedentarismo $(60 \%)$, permitiu-se detectar uma razão de prevalência de 2,0 como estatisticamente significativa, com poder de $80 \%$ e nível de significância de 95\%.

O processo amostral foi estratificado em três estágios: o tipo da escola (estadual, municipal e particular), a série de ensino (da $5^{\mathrm{a}}$ série do Ensino Fundamental ao $3^{\circ}$ ano do Ensino Médio) e o setor do município. A quantidade de escolas foi determinada proporcionalmente à quantidade de cada tipo, por setor do município, tendo sido selecionadas cinco escolas estaduais, cinco municipais e duas particulares. Duas escolas recusaram-se a participar do estudo, tendo sido incluídas por sorteio duas novas escolas com características parecidas às anteriores, respeitando o tipo de escola e o setor do município. Após, sorteou-se, em cada escola, uma quantidade de turmas para atingir o número de sujeitos que representasse o setor, o tipo de escola e a série de ensino, totalizando 75 turmas (aleatório por conglomerados).

No primeiro dia foi entregue aos escolares o termo de consentimento livre e esclarecido, o qual deveria ser devolvido no dia seguinte. Os escolares que retornavam o termo de consentimento respondiam o questionário. A aplicação do questionário foi realizada por acadêmicos e profissionais de Educação Física e Nutrição devidamente treinados. O questionário incluiu questões sobre: sexo, idade, quantidade de pessoas que residiam na moradia, escolaridade do chefe da família, possuir TV no quarto, forma de deslocamento para a escola, atividade física do pai e da mãe, nível de atividade física (NAF) 
e número de horas diárias e dias por semana assistindo TV, jogando videogame e utilizando o computador.

Para o NAF, utilizou-se uma adaptação ${ }^{14}$ do instrumento de auto-recordação proposto por Bouchard et $\mathrm{al}^{15}$. A partir de instruções prévias, os avaliados preencheram o diário de atividade física, registrando as atividades realizadas durante três dias da semana, sendo um dia do finalde-semana (domingo) e dois dias da semana (um mais ativo e outro menos ativo). Para isso, cada dia foi dividido em 36 períodos de 30 minutos cada (das $6 \mathrm{~h}$ até as $24 \mathrm{~h}$ do dia) e, para cada período de 30 minutos, o sujeito identificava na escala de atividades de 1 a 9, o tipo de atividade física praticada naquele espaço de tempo. Para a categorização do NAF dos adolescentes, fez-se a média ponderada do gasto energético dos três dias da semana. Considerou-se como praticantes de baixo NAF os adolescentes que apresentaram gasto energético menor que $37 \mathrm{kcal} / \mathrm{kg} / \mathrm{dia}^{17}$. Os demais adolescentes foram considerados como praticantes de níveis adequados de atividade física.

O número de horas semanais que os escolares afirmavam usualmente passar assistindo TV, jogando videogame e utilizando o computador foi somado e considerou-se como exposição elevada a comportamentos sedentários (CSE) a realização destas atividades por 14 horas ou mais por semana $\left(2 \mathrm{~h} / \mathrm{dia}^{16}\right)$. A idade dos adolescentes foi agrupada em duas faixas etárias: 11-14 anos e 15-17 anos.

Foi realizado um estudo piloto com 122 sujeitos de uma escola que não foi incluída na amostra, no qual se verificou a reprodutibilidade do questionário. O número de horas em comportamentos sedentários apresentou reprodutibilidade (coeficiente de correlação intraclasse) de 0,92 , sendo a menor aos 11 anos $(0,85)$. O gasto energético (NAF) apresentou reprodutibilidade de 0,88 , sendo menor aos 12 anos $(0,73)$ e maior aos $17(0,94)$. Após todos os questionários serem digitados, um dos pesquisadores conferiu os dados de todos os questionários para verificar possíveis erros de digitação, corrigindo-os quando necessário.

O teste qui-quadrado foi empregado para comparar entre os sexos a proporção de adolescentes com baixo nível de atividade física (baixo NAF), exposição elevada a comportamentos sedentários (CSE), que residiam em até 4 pessoas na moradia, com escolaridade do chefe da família maior que 9 anos de estudo, que possuíam TV no quarto, deslocavam-se passivamente à escola e relatavam baixo NAF do pai e da mãe. Também foi utilizado para verificar, por sexo, a proporção de adolescentes com baixo NAF e CSE nos subgrupos dos fatores associados.

A razão de prevalência $(\mathrm{RP})$ e os respectivos intervalos de confiança $\left(\mathrm{IC}_{95 \%}\right)$ foram empregados, por meio da regressão de Poisson, para analisar a associação (análise bruta e ajustada) entre as variáveis dependentes (NAF e comportamentos sedentários) e as independentes (faixa etária - 11-14 anos / 15-17; residem na moradia - 5 ou mais pessoas / até 4; escolaridade do chefe da família - até 8 anos / 9 ou mais; apresentar TV no quarto - não / sim; deslocamento para a escola - passivo (carro, moto ou ônibus) / ativo (a pé ou bicicleta para ir à escola, voltar ou ambos); e atividade física do pai e da mãe - 3 dias ou mais por semana / até 2 dias (60 minutos ou mais por dia)).

Foram utilizados dois modelos na análise ajustada. No primeiro (estratificado por sexo), utilizou-se como variável dependente o NAF, considerando os adolescentes com gasto energético menor que $37 \mathrm{kcal} / \mathrm{kg} / \mathrm{dia}$ (baixo NAF) como grupo de referência. No segundo modelo (estratificado por sexo), os comportamentos sedentários foram utilizados como variável dependente, sendo considerados os adolescentes com valores maiores do que 14 horas/semana assistindo TV, jogando videogame e utilizando o computador (CSE) como grupo de referência. Utilizou-se modelo hierarquizado em três níveis, participando do primeiro nível as variáveis sociodemográficas (faixa etária, número de pessoas que residem na moradia e escolaridade do chefe da família); do segundo nível, possuir TV no quarto e forma de deslocamento para a escola; e do terceiro nível, as variáveis de comportamento dos pais (atividade física do pai e da mãe). Permaneceram no modelo a faixa etária e as variáveis que apresentaram $p<0,25$. Para todas as análises utilizou-se $\mathrm{p}<0,05\left(\mathrm{IC}_{95 \%}\right)$.

\section{RESULTADOS}

Comparação de freqüências percentuais e absolutas das variáveis dependentes e dos fatores associados entre rapazes e moças de Caxias do Sul, RS, são mostrados na tabela 1 .

A comparação entre os sexos evidenciou que os rapazes apresentaram maiores preva- 
Tabela 1. Comparação de freqüências percentuais e absolutas das variáveis dependentes e dos fatores associados entre rapazes e moças de Caxias do Sul, RS. 2007.

\begin{tabular}{lcccc}
\hline Variáveis & $\mathrm{n}$ & $\begin{array}{c}\text { Geral } \\
\%(\mathrm{n})\end{array}$ & $\begin{array}{c}\text { Rapazes } \\
\%(\mathrm{n})\end{array}$ & $\begin{array}{c}\text { Moças } \\
\%(\mathrm{n})\end{array}$ \\
\hline Baixo NAF (\%) & 1634 & $55,8(911)$ & $43,2(331)$ & $66,8(580)^{*}$ \\
CSE (\%) & 1573 & $86,6(1363)$ & $89,6(664)$ & $84,0(699)^{*}$ \\
Residem em até 4 pessoas na moradia (\%) & 1621 & $67,0(1086)$ & $67,6(506)$ & $66,5(580)$ \\
Escolaridade 9 anos ou mais chefe família (\%) & 1524 & $64,9(989)$ & $63,8(448)$ & $65,8(541)$ \\
Possuem TV no quarto (\%) & 1655 & $46,6(772)$ & $52,4(404)$ & $41,6(368)^{*}$ \\
Deslocamento passivo à escola (\%) & 1597 & $38,1(609)$ & $34,6(258)$ & $41,2(351)^{*}$ \\
Atividade física da mãe <3 dias/semana (\%) & 1640 & $72,6(1190)$ & $75,8(582)$ & $69,7(608)^{*}$ \\
Atividade física do pai <3 dias/semana (\%) & 1589 & $65,8(1046)$ & $65,4(494)$ & $66,2(552)$ \\
\hline
\end{tabular}

*p<0,05 no teste qui-quadrado entre os rapazes e as moças. NAF: nível de atividade física; CSE: exposição elevada a comportamentos sedentários.

Tabela 2. Freqüência de rapazes e moças com baixo nível de atividade física (baixo NAF) e exposição elevada a comportamentos sedentários (CSE) a partir dos fatores associados em adolescentes de Caxias do Sul, RS. 2007.

\begin{tabular}{|c|c|c|c|c|c|c|c|c|}
\hline \multirow{3}{*}{ Variáveis } & \multicolumn{4}{|c|}{ Rapazes } & \multicolumn{4}{|c|}{ Moças } \\
\hline & \multicolumn{2}{|c|}{ Baixo NAF } & \multicolumn{2}{|c|}{ CSE } & \multicolumn{2}{|c|}{ Baixo NAF } & \multicolumn{2}{|c|}{ CSE } \\
\hline & $\%(n)$ & $\mathrm{p}$ & $\%(n)$ & $\mathrm{p}$ & $\%(n)$ & p & $\%(n)$ & $\mathrm{p}$ \\
\hline \multicolumn{9}{|l|}{ Faixa etária } \\
\hline $11-14$ anos & $42,6(196)$ & 0,680 & $89,5(393)$ & 0,925 & $58,2(276)$ & $<0,001$ & $83,0(381)$ & 0,379 \\
\hline 15-17 anos & $44,1(135)$ & & $89,7(271)$ & & $77,2(304)$ & & 85,3 (318) & \\
\hline \multicolumn{9}{|l|}{ Residem na moradia } \\
\hline 5 ou mais pessoas & $38,0(90)$ & $0,044^{*}$ & $87,9(203)$ & 0,247 & $60,2(171)$ & $0,004^{*}$ & $81,1(214)$ & 0,092 \\
\hline Até 4 pessoas & $45,9(227)$ & & 90,7 (438) & & 70,1 (397) & & $85,7(472)$ & \\
\hline \multicolumn{9}{|l|}{ Escolaridade ch fam } \\
\hline Até 8 anos & $40,8(100)$ & 0,298 & $88,8(213)$ & 0,487 & 70,8 (196) & $0,036^{*}$ & 79,8 (209) & $0,022 *$ \\
\hline 9 anos ou mais & 44,9 (199) & & $90,4(388)$ & & $63,4(334)$ & & $86,2(442)$ & \\
\hline \multicolumn{9}{|l|}{ TV no quarto } \\
\hline Não & $43,3(157)$ & 0,950 & $87,9(305)$ & 0,116 & $64,7(325)$ & 0,183 & $79,0(376)$ & $<0,001^{*}$ \\
\hline $\operatorname{Sim}$ & $43,5(170)$ & & $91,4(352)$ & & $69,1(248)$ & & 90,6 (318) & \\
\hline \multicolumn{9}{|c|}{ Deslocamento escola } \\
\hline Ativo & $43,5(207)$ & 0,712 & $88,9(409)$ & 0,473 & $62,2(301)$ & $0,001^{*}$ & $82,8(384)$ & 0,422 \\
\hline Passivo & $42,1(106)$ & & $90,7(223)$ & & $73,1(253)$ & & $84,9(281)$ & \\
\hline \multicolumn{9}{|l|}{ Atividade física mãe } \\
\hline 3 ou mais dias/sem & $37,9(69)$ & 0,120 & $88,8(159)$ & 0,659 & $63,8(164)$ & 0,191 & $84,5(213)$ & 0,896 \\
\hline Até 2 dias/sem & $44,5(253)$ & & $90,0(494)$ & & $68,4(407)$ & & $84,2(473)$ & \\
\hline \multicolumn{9}{|l|}{ Atividade física pai } \\
\hline 3 ou mais dias/sem & $40,7(103)$ & 0,319 & $89,5(222)$ & 0,782 & $64,6(179)$ & 0,559 & $83,3(220)$ & 0,623 \\
\hline Até 2 dias/sem & $44,5(216)$ & & $90,2(422)$ & & $66,7(358)$ & & $84,7(437)$ & \\
\hline
\end{tabular}

${ }^{*} \mathrm{p}<0,05$ no teste qui-quadrado.

lências de CSE ( $\mathrm{p}=0,001)$, de TV no quarto $(\mathrm{p}<0,001)$ e de mães que praticavam pouca atividade física $(\mathrm{p}=0,006)$ do que as moças, enquanto estas obtiveram maior prevalência de baixo $\operatorname{NAF}(\mathrm{p}<0,001)$ e deslocamento passivo à escola $(\mathrm{p}=0,007)$.

A prevalência de baixo NAF e de CSE a partir dos fatores associados foi analisada separadamente por sexo (Tabela 2). Nos rapazes, observou-se maiores prevalências de baixo NAF nos que residiam com até 4 pessoas, em relação aos que residiam com 5 ou mais pessoas, enquanto que no CSE não se verificou diferenças significativas em nenhum dos fatores analisados. Para as moças, houve maiores prevalências de baixo NAF na faixa etária de 15-17 anos, nas que residiam com até 4 pessoas, o chefe da família possuia escolaridade de até 8 anos e realizavam deslocamento passivo à escola, em relação aos grupos de referência. Ocorreu maior prevalência de CSE nas moças com escolaridade do chefe da família elevada e nas que possuíam TV no quarto, em relação aos grupos de referência.

Nos rapazes, o baixo NAF associou-se, na análise bruta, a residir com, até quatro pessoas 
Tabela 3. Razões de prevalência brutas e ajustadas, utilizando como variáveis dependentes baixo nível de atividade física (baixo NAF) e exposição elevada a comportamentos sedentários (CSE) em rapazes de Caxias do Sul, RS. 2007.

\begin{tabular}{|c|c|c|c|c|c|c|c|c|}
\hline \multirow{2}{*}{ Variáveis (nivel hierárquico) } & \multicolumn{4}{|c|}{ Baixo NAF } & \multicolumn{4}{|c|}{ CSE } \\
\hline & $\mathrm{RP}_{\mathrm{B}}$ & $\mathrm{IC}_{95 \%}$ & $\mathrm{RP}_{\mathrm{A}}$ & $\mathrm{IC}_{95 \%}$ & $\mathrm{RP}_{\mathrm{B}}$ & $\mathrm{IC}_{95 \%}$ & $\mathrm{RP}_{\mathrm{A}}$ & $\mathrm{IC}_{95 \%}$ \\
\hline \multicolumn{9}{|l|}{ Faixa etária $_{(1)}$} \\
\hline $11-14$ anos & 1 & & 1 & & 1 & & 1 & \\
\hline $15-17$ anos & 1,04 & $0,88-1,22$ & 1,02 & $0,86-1,21$ & 1,00 & $0,95-1,95$ & 0,99 & $0,94-1,04$ \\
\hline \multicolumn{9}{|l|}{ Residem na moradia $_{(1)}$} \\
\hline 5 ou mais pessoas & 1 & & 1 & & 1 & & & \\
\hline Até 4 pessoas & 1,21 & $1,00-1,46$ & 1,21 & $1,00-1,46$ & 1,03 & $0,98-1,09$ & & \\
\hline \multicolumn{9}{|l|}{ Escolaridade chefe fam } \\
\hline Até 8 anos & 1 & & & & 1 & & & \\
\hline 9 anos ou mais & 1,10 & $0,92-1,32$ & & & 1,02 & $0,96-1,08$ & & \\
\hline \multicolumn{9}{|l|}{ TV no quarto ${ }_{(2)}$} \\
\hline Não & 1 & & & & 1 & & 1 & \\
\hline Sim & 1,01 & $0,85-1,18$ & & & 1,04 & 0,99-1,09 & 1,04 & 0,99-1,09 \\
\hline \multicolumn{9}{|l|}{ Deslocamento à escola $_{(2)}$} \\
\hline Ativo & 1 & & & & 1 & & & \\
\hline Passivo & 0,97 & $0,81-1,16$ & & & 1,02 & $0,97-1,07$ & & \\
\hline \multicolumn{9}{|l|}{ Atividade física da mãe $_{(3)}$} \\
\hline 3 ou mais dias/semana & 1 & & & & 1 & & & \\
\hline Até 2 dias/semana & 1,17 & $0,95-1,44$ & 1,23 & $1,00-1,53$ & 1,01 & $0,95-1,07$ & & \\
\hline \multicolumn{9}{|l|}{ Atividade física do pai ${ }_{(3)}$} \\
\hline 3 ou mais dias/semana & 1 & & & & 1 & & & \\
\hline Até 2 dias/semana & 1,09 & $0,91-1,31$ & & & 1,01 & $0,96-1,06$ & & \\
\hline
\end{tabular}

$\mathrm{RP}_{\mathrm{B}}$ : razão de prevalência bruta; $\mathrm{RP}_{\mathrm{A}}$ : razão de prevalência ajustada pela faixa etária e pelas variáveis com $\mathrm{p}<0,25$.

Tabela 4. Razões de prevalência brutas e ajustadas, utilizando como variáveis dependentes baixo nível de atividade física (baixo NAF) e exposição elevada a comportamentos sedentários (CSE) em moças de Caxias do Sul, RS. 2007.

\begin{tabular}{|c|c|c|c|c|c|c|c|c|}
\hline \multirow{2}{*}{ Variáveis (nivel hierárquico) } & \multicolumn{4}{|c|}{ Baixo NAF } & \multicolumn{4}{|c|}{ CSE } \\
\hline & $\mathrm{RP}_{\mathrm{B}}$ & $\mathrm{IC}_{95 \%}$ & $\mathrm{RP}_{\mathrm{A}}$ & $\mathrm{IC}_{95 \%}$ & $\mathrm{RP}_{\mathrm{B}}$ & $\mathrm{IC}_{95 \%}$ & $\mathrm{RP}_{\mathrm{A}}$ & $\mathrm{IC}_{95 \%}$ \\
\hline \multicolumn{9}{|l|}{ Faixa etária } \\
\hline 11-14 anos & 1 & & 1 & & 1 & & 1 & \\
\hline 15-17 anos & 1,33 & $1,21-1,45$ & 1,30 & $1,18-1,44$ & 1,03 & $0,97-1,09$ & 1,04 & $0,98-1,11$ \\
\hline \multicolumn{9}{|l|}{ Residem na moradia $_{(1)}$} \\
\hline 5 ou mais pessoas & 1 & & 1 & & 1 & & 1 & \\
\hline Até 4 pessoas & 1,16 & $1,04-1,30$ & 1,17 & $1,04-1,31$ & 1,06 & $0,99-1,13$ & 1,07 & $0,99-1,15$ \\
\hline \multicolumn{9}{|l|}{ Escolaridade chefe fam } \\
\hline Até 8 anos & 1 & & 1 & & 1 & & 1 & \\
\hline 9 anos ou mais & 0,90 & $0,81-0,99$ & 0,92 & $0,84-1,02$ & 1,08 & $1,01-1,16$ & 1,08 & $1,01-1,16$ \\
\hline \multicolumn{9}{|l|}{ TV no quarto $(2)$} \\
\hline Não & 1 & & 1 & & 1 & & 1 & \\
\hline Sim & 1,07 & $0,97-1,17$ & 1,13 & $1,02-1,25$ & 1,15 & $1,08-1,21$ & 1,15 & $1,08-1,22$ \\
\hline \multicolumn{9}{|l|}{ Deslocamento à escola $_{(2)}$} \\
\hline Ativo & 1 & & 1 & & 1 & & & \\
\hline Passivo & 1,18 & $1,07-1,29$ & 1,10 & $1,00-1,22$ & 1,03 & $0,96-1,09$ & & \\
\hline \multicolumn{9}{|l|}{ Atividade física da mãe ${ }_{(3)}$} \\
\hline 3 ou mais dias/semana & 1 & & 1 & & 1 & & & \\
\hline Até 2 dias/semana & 1,07 & $0,96-1,19$ & 1,07 & $0,95-1,20$ & 1,00 & $0,93-1,06$ & & \\
\hline \multicolumn{9}{|l|}{ Atividade física do pai ${ }_{(3)}$} \\
\hline 3 ou mais dias/semana & 1 & & & & 1 & & & \\
\hline Até 2 dias/semana & 1,03 & $0,93-1,15$ & & & 1,02 & $0,95-1,08$ & & \\
\hline
\end{tabular}

$\mathrm{RP}_{\mathrm{B}}$ : razão de prevalência bruta; $\mathrm{RP}_{\mathrm{A}}$ : razão de prevalência ajustada pela faixa etária e pelas variáveis com $\mathrm{p}<0,25$.

(Tabela 3). A análise ajustada, por sua vez, indicou associação do baixo NAF com residir com até 4 pessoas e com a atividade física da mãe até 2 dias por semana.

Nas moças, baixo NAF associou-se, na análise bruta, com a faixa etária de 15-17 anos, 
residir em até 4 pessoas, menor escolaridade do chefe da família e deslocamento passivo à escola (Tabela 4). A análise ajustada indicou associação do baixo NAF, no primeiro nível, com a faixa etária de 15-17 anos e residir com até 4 pessoas. No segundo nível, baixo NAF associou-se, mesmo após ajustado para a faixa etária, número de pessoas que residiam na moradia e escolaridade do chefe da família, com possuir TV no quarto e deslocar-se passivamente à escola. $\mathrm{O}$ CSE das moças associou-se, na análise bruta, à faixa etária de 15-17 anos, à maior escolaridade do chefe da família e à presença de TV no quarto. A análise ajustada indicou associação do CSE, no primeiro nível, com a escolarização elevada do chefe da família e, no segundo nível, com possuir TV no quarto.

\section{DISCUSSÃO}

Neste estudo, os rapazes apresentaram maior prevalência de CSE do que as moças, enquanto estas obtiveram maior prevalência de baixo NAF. A literatura é consistente em relação às moças apresentarem maior prevalência de baixo NAF, o que já foi amplamente discutido ${ }^{4,12,13}$. Os rapazes usualmente passam maior número de horas em comportamentos sedentários do que as moças ${ }^{18,19}$. Porém esta associação parece diferir entre os tipos de comportamentos sedentários (TV, videogame e computador). Em adolescentes norte-americanos, não foi encontrada associação entre o sexo e o número de horas semanais assistindo $\mathrm{TV}^{20}$, entretanto, em outro estudo, encontrou-se associação dos rapazes com o uso do computador ${ }^{21}$.

Nas moças, verificou-se maior prevalência de baixo NAF na faixa etária de 15-17 anos. Durante a adolescência, estudos têm reportado diminuição dos níveis de atividade física com a idade $^{22,23}$, principalmente nas moças ${ }^{18,24}$, apesar de esta associação nem sempre ser encontrada ${ }^{25}$. A diminuição da atividade física nesta fase da vida, normalmente, acarreta em maiores níveis de sedentarismo na fase adulta ${ }^{2,25}$, o que é um fator de risco para doenças cardiovasculares e metabólicas ${ }^{6,7}$.

Encontrou-se maior prevalência de TV no quarto nos rapazes, como observado em adolescentes europeus ${ }^{26}$. No entanto, este fator apresentou associação com o baixo NAF e o CSE somente nas moças. Em rapazes australianos, verificou-se associação da atividade física moderada e vigorosa com possuir TV no quarto, enquanto nas moças, não se verificou associação com a atividade física total ou de intensidades distintas. Os autores sugeriram que possuir TV no quarto estava associado ao nível socioeconômico mais elevado, no qual os sujeitos praticavam mais atividade física ${ }^{27}$.

O número de pessoas residindo na mesma moradia é uma medida socioeconômica, e apresentou associação positiva com o NAF. Em adolescentes norte-americanos ${ }^{28}$ e australianos ${ }^{29}$ não foi observada associação. No entanto, de modo geral, o NAF tem apresentado associação positiva com características socioeconômicas em adolescentes ${ }^{8,9}$, o que ocorreu de modo contrário neste estudo.

A escolaridade do chefe da família apresentou associação positiva com o CSE nas moças. Em adolescentes chineses ${ }^{30}$ e rapazes norte-americanos ${ }^{21,25}$ não foram encontradas associações. Moças norte-americanas apresentaram esta associação de forma inversa ${ }^{25}$, assim como adolescentes belgas para escolaridade dos pais e tempo de $\mathrm{TV}^{31}$. Pais com maior escolaridade, supostamente, têm maior conhecimento da importância de um estilo de vida ativo. No entanto, os achados divergentes deste estudo podem ser justificados pela possibilidade de os pais com maior escolaridade terem mais condições financeiras de comprar aparelhos eletrônicos.

O deslocamento passivo à escola associouse ao baixo NAF nas moças, sendo que estas obtiveram maior prevalência de deslocamento passivo do que os rapazes. Em adolescentes, esta associação é bastante consistente ${ }^{10,30}$, principalmente nas moças ${ }^{32,33}$. O deslocamento ativo à escola tem sido amplamente adotado como estratégia no combate à obesidade e na manutenção de um estilo de vida ativo ${ }^{34}$.

$\mathrm{O}$ baixo NAF dos rapazes apresentou associação com o baixo NAF da mãe, sendo que estes apresentaram maior prevalência de mães inativas. De modo geral, o baixo NAF tem sido associado com a inatividade física dos pais ${ }^{8,9}$. Estudo em adolescentes australianos indicou esta associação em 1985. No entanto, ao analisar as mesmas variáveis em 1997-99, não se encontrou esta relação, sugerindo que mudanças sociais, como a falta de estrutura familiar e as escolhas do tipo de exercício pelos pais limitaram as oportunidades de a família praticar atividades físicas conjuntamente ${ }^{35}$, fato que neste estudo 
possivelmente tenha ocorrido nas moças e nas associações com a atividade física do pai.

Por ser um estudo de corte transversal, devese levar em consideração a possibilidade de ter ocorrido causalidade reversa nas associações entre as variáveis dependentes e possuir TV no quarto, forma de deslocamento à escola e atividade física do pai e da mãe. $O$ instrumento utilizado para a mensuração do NAF não foi submetido a um processo de validação. No entanto, verificou-se, em todas as idades incluídas no estudo, que a reprodutibilidade do instrumento foi muito boa. Deve-se ressaltar, ainda, que a comparação entre os estudos presente nesta discussão deve ser compreendida com cautela, em decorrência dos diferentes métodos de mensuração da atividade física e critérios operacionais de baixo NAF existentes na literatura.

\section{CONCLUSÃO}

Nos adolescentes de Caxias do Sul, RS, o baixo NAF apresentou maior número de fatores associados do que o CSE, assim como as moças em relação aos rapazes. $\mathrm{O}$ aumento dos níveis de atividade física nas moças deve focar principalmente os fatores: faixa etária de 15-17 anos, residir com até 4 pessoas, possuir TV no quarto e deslocar-se passivamente à escola; e nos rapazes, residir com até 4 pessoas e baixo NAF da mãe. A diminuição dos comportamentos sedentários nas moças deve focar os fatores: escolaridade elevada do chefe da família e possuir TV no quarto.

Este estudo contribuiu, principalmente, ao relatar associação entre as medidas de aproximação socioeconômica (escolaridade elevada do chefe da família e residir com até 4 pessoas) e os comportamentos de risco (baixo NAF e CSE), fato que a maioria dos estudos tem apresentado de forma inversa. O conhecimento dos fatores associados ao baixo NAF e CSE na população específica facilita a implantação de intervenções mais efetivas, a fim de promover um estilo de vida mais ativo.

\section{REFERÊNCIAS BIBLIOGRÁFICAS}

1. Twisk JWR. Physical activity guidelines for children and adolescents: a critical review. Sports Med 2001;31(8):617-627.

2. Hallal PC, Victora CG, Azevedo MR, Wells JCK. Adolescent physical activity and health: a systematic review. Sports Med 2006;36(12):1019-1030.
3. Mendes MJFL, Alves JGB, Alves AV, Siqueira PP, Freire EFC. Associação de fatores de risco para doenças cardiovasculares em adolescentes e seus pais. Rev Bras Saúde Mat Inf 2006;6(S1):49-54.

4. Silva RCR, Malina RM. Nível de atividade física em adolescentes do Município de Niterói, Rio de Janeiro, Brasil. Cad Saúde Pública 2000;16(4):1091-1097.

5. Twisk JWR, Kemper HCG, Van Mechelen W, Post GB. Clustering of Risk Factors for Coronary Heart Disease: The Longitudinal Relationship with Lifestyle. AEP 2001;11(3): 157-165.

6. Ribeiro JC, Guerra S, Oliveira J, Teixeira-Pinto A, Twisk JWR, Duarte JA et al. Physical activity and biological risk factors clustering in pediatric population. Prev Med 2004;39:596-601.

7. Andersen LB, Harro M, Sardinha LB, Froberg K, Ekelund U, Brage S et al. Physical activity and clustered cardiovascular risk in children: a crosssectional study (The European Youth Heart Study). Lancet 2006;368(9532):299-304.

8. Sallis JF, Prochaska JJ, Taylor WC. A review of correlates of physical activity of children and adolescents. Med Sci Sports Exerc 2000;32(5):963-975.

9. Tudor-Locke C, Neff LJ, Ainsworth BE, Addy CL, Popkin BM. Omission of active commuting to school and the prevalence of children's health related physical activity levels: the Russian Longitudinal Monitoring Study. Child: Care, Health Develop 2002;28(6):507-512.

10. Horst KVD, Paw MJCA, Twisk JWR, Mechelen WV. A brief review on correlates of physical activity and sedentariness in youth. Med Sci Sports Exerc 2007;39(8):1241-1250.

11. Luiz RR, Magnanini MMF. A lógica da determinação do tamanho da amostra em investigações epidemiológicas. Cad Saúde Coletiva 2000;8(2): 9-28.

12. Farias Jr. JC, Lopes AS. Comportamentos de risco relacionados à saúde em adolescentes. Rev Bras Ciên Mov 2004;12(1):7-12.

13. Hallal PC, Bertoldi AD, Gonçalves H, Victora CG. Prevalência de sedentarismo e fatores associados em adolescentes de 10-12 anos de idade. Cad Saúde Pública 2006;22(6):1277-1287.

14. Farias Jr. JC, Pires MC, Lopes, AS. Reprodutibilidade de um questionário para o levantamento de informações sobre comportamentos relacionados à saúde em adolescentes. Rev Bras Ciên Mov 2002;10(3):43-48.

15. Bouchard C, Tremblay A, Le Blanc C, Lortie G, Sauard R, Therial G. A method to assess energy expenditure in children and adults. Am J Clin Nutr 1983;37(3):461-467.

16. American Academy of Pediatrics. Children, adolescents, and television. Pediatrics 2001;107(2):423-6.

17. Cale L. Self-report measures of children's physical activity: recommendations for future development and a new alternative measure. Health Educ J 1994;53(4):439-453. 
18. Sallis JF, Zakarian JM, Hovell MF, Hofstetter CR. Ethnic, socioeconomic, and sex differences in physical activity among adolescents. J Clin Epidemiol 1996;49(2):125-134.

19. Guedes DP, Guedes JERP, Barbosa DS, Oliveira JA. Níveis de prática de atividade física habitual em adolescentes. Rev Bras Med Esporte 2001;7(6):187-199.

20. McGuire MT, Hannan PJ, Neumark-Szteiner D, Cossrow NHF, Story M. Parental correlates of physical activity in a racially/ethnically diverse adolescent sample. J Adolesc Health 2002;30(4):253-261.

21. Norman GJ, Schmid BA, Sallis JF, Calfas KJ, Patrick K. Psychosocial and environmental correlates of adolescent sedentary behaviors. Pediatrics 2005;116(4):906-916.

22. Neumark-Szteiner D, Story M, Hannan PJ, Tharp T, Rex J. Factors associated with changes in physical activity: a cohort study of inactive adolescent girls. Arch Pediatr Adolesc Med2003;157(8):803-10.

23. Nelson MC, Gordon-Larsen P, Adair LS, Popkin BM. Adolescent Physical activity and sedentary behavior: patterning and long-term maintenance. Am J Prev Med 2005;28(3):259-266.

24. Strauss RS, Rodzilsky D, Burack G, Colin M. Psychosocial correlates of physical activity in healthy children. Arch Pediatr Adolesc Med 2001;155(4): 897-902.

25. Schmitz KH, Lytle LA, Phillips GA, Murray DM, Birnbaum AS, Kubik MY. Psychosocial correlates of physical activity and sedentary leisure habits in young adolescents: The Teens Eating for Energy and Nutrition at School Study. Prev Med 2002;34(2):266-278.

26. Roe K. Adolescents` media use: a European view. J Adolesc Health 2000;27(2 suppl):15-21.

27. Hume C, Salmon J, Ball K. Children perceptions of their home and neighborhood environments, and their association with objectively measured physical activity: a quantitative and qualitative study. Health Educ Res 2005;20(1):1-13.

28. Zakarian JM, Hovell MF, Hofstetter CR, Sallis JF, Keating KJ. Correlates of vigorous exercise in a predominantly low SES and minority high school population. Prev Med 1994;23(3):314-321.
29. Spinks A, Macpherson A, Bain C, McClure R. Determinants of sufficient daily activity in Australian primary school children. J Paed Child Health 2006;42(11):674-679.

30. Shi Z, Lien N, Kumar BN, Holmboe-Ottesen G. Physical activity and associated socio-demographic factors among school adolescents in Jiangsu Province, China. Prev Med 2006;43(3):218-221.

31. Vereecken CA, Maes L, Bacquer DD. The influence of parental occupation and the pupils' educational level on lifestyle behaviors among adolescents in Belgium. J Adolesc Health 2004;34(4):330-338.

32. Cooper AR, Andersen LB, Wedderkopp N, Page AS, Froberg K. Physical activity levels of children who walk, cycle, or are driven to school. Am J Prev Med 2005;29(3):179-184.

33. Tudor-Locke C, Ainsworth BE, Popkin BM. Active commuting to school: An overlooked source of childrens physical activity? Sports Med 2001;31(5):309-313.

34. Martin M, Dollman J, Norton K, Robertson I. A decrease in the association between the physical activity patterns of Australian parents and their children; 1985-1997. J Sci Med Sport 2005;8(1):71-76.

\section{Agradecimentos}

Aos acadêmicos e professores dos cursos de Educação Física e Nutrição da Universidade de Caxias do Sul (UCS), pela ajuda na coleta de dados. Aos professores José Cazuza de Farias Junior e Kelly Samara da Silva, pelas contribuições para a concepção deste artigo.

\section{Endereço para correspondência}

Rua Vereador José do Vale Pereira, 48/204

88080-240, Florianópolis-SC, Brasil

E-mail: dgvasques@hotmail.com 